\title{
7. MÉCANIQUE CÉLESTE (CELESTIAL MECHANICS)
}

PRESIDENT : J. Henrard

VICE-PRESIDENT : A. Deprit

ORGANIZING COMMITTEE : K.B. Bhatnagar, V.A. Brumberg, J. Chapront, S. Ferraz-Mello, C. Froeschlé, J.D. Hadjidemetriou, He Miaofu, K.V. Kholshevnikov, H. Kinoshita, A. Milani, A.E. Roy, P.K. Seidelmann.

\section{A. Introduction}

During 1988-1990 Commission 7 has sponsored or co-sponsored several IAU conferences: Colloquium No. 109 "Application of Computer Technology to Dynamical Astronomy" (Gaithersburg, July 1988), Symposium No. 141 "Inertial Coordinate System on the Sky" (Pulkovo, October 1989), Colloquium No. 127 "Reference Frames" (Virginia Beach, October 1990), Colloquium No. 132 "Instability, Chaos and Predictability in Celestial Mechanics and Stellar Systems" (Delhi, October 1990). The colloquium No. 118 "Dynamics of Small Bodies in the Solar System" which was to be held in Nanjing in June 1989 had unfortunately to be postponed then cancelled. Other meetings of interest to the members of Commission 7 were the $2^{\text {nd }}$ Alexander von Humbolt Colloquium on "Long Term Evolution of Planetary Systems" (Ramsau, March 1988), the Colloquium "Asteroids, Comets, Meteors III" (Uppsala, June 1989), the colloquium "Mécanique Céleste et Systèmes Hamiltoniens" (Luminy, May 1990) and the NATO Advanced Study Institute on "Predictability, Stability and Chaos in N-Body Dynamical Systems" (Cortina d'Ampezzo, August 1990).

Several regional meetings were also held during the past three years: the Division of Dynamical Astronomy of the American Astronomical Society met at Gaithersburg (July 1988), at Pasadena (August 1989) and at Austin (April 1990). The Japanese Celestial Mechanicians held their $22^{\text {nd }}$ Symposium in Tokyo in January 1989 and their $23^{\text {rd }}$ Symposium in Kyoto in December 1989. Our Chinese colleagues organized symposia of the Chinese Astronomical Society on "Frontiers of Celestial Mechanics" (Jiande, May 1988, Symposium no 77) and on "Relativity in Astrometry, Celestial Mechanics and Geodesy" (Shengsi, August 1989, Symposium no 90). Our French colleagues organized (with a large participation from over all Europe) a Spring School on "Méthodes Modernes en Mécanique Céleste- théorie des perturbations et chaos intrinsèque" in Goutelas (April 1989) and the "Deuxième Table Ronde de Planétologie Dynamique" in Vars (March 1990).

Several books were published during these years:

J. Danby: $2^{\text {nd }}$ and revised edition of "Fundamental of Celestial Mechanics", Willmann Bell 1988.

A.E. Roy: $3^{\text {rd }}$ and revised edition of "Orbital Motion", Adam Hilger 1988.

V.I. Arnold (ed.): "Dynamical Systems III", Springer Verlag 1988.

V.A. Antonov, E.I. Timoshkova and K.V. Khoshevnikov: "Introduction to Newtonian Potential Theory" Nauka, Moscou 1988 (in Russian).

V. Szebehely: "Adventure in Celestial Mechanics", Univ. of Texas Press 1989.

M.E. Soffel: "Relativity in Astrometry, Celestial Mechanics and Geodesy", Springer Verlag 1989. 
J. Kovalevsky, I.I. Mueller and B. Kolaczek (eds.): "Reference Frames in Astronomy and Geophysics" Kluwer A.P. 1989.

R.P. Binzel (ed.): “Asteroids II" Univ. of Arizona Press 1989.

A.D. Bruno: "Local Methods in Nonlinear Differential Equations" Springer Verlag 1989.

Zheng Xuetang and Ni Caixia: "Celestial Mechanics and Astrodynamics" Beijing Normal Univ. Press 1989.

J. Kovalevsky: "Astrométrie Moderne" Springer Verlag 1990.

B. Bertotti and P. Farinella: "Physics of the Earth and the Solar System" Kluwer A.P. 1990.

A.D. Bruno: "The Restricted Three-Body Problem" Nauka, Moscou 1990 (in Russian).

Ch. Marchal: "The Three-Body Problem" Elsevier 1990.

V.A. Brumberg: "Essential Relativistic Celestial Mechanics"” Adam Hilger in press.

Let us mention also the publication of proceedings of conferences:

R. Dvorak and J. Henrard (eds.): "Long Term Evolution of Planetary Systems" Kluwer A.P. 1988 (reprinted from volume 43 of Celestial Mechanics).

A.E. Roy (ed.): "Long-term Dynamical Behaviour of Natural and Artificial N-Body Systems" Kluwer A.P. 1988.

M.J. Valtonen (ed.): "The Few Body Problem" Kluwer A.P. 1988.

P.K. Seidelmann and J. Kovalevsky (eds.): "Application of Computer Technology to Dynamical Astronomy" Kluwer A.P. 1989 (reprinted from volume 45 of Celestial Mechanics).

C.I. Lagerkvist et al. (eds.): "Asteroids, Comets, Meteors III" Uppsala Observatory 1990.

D. Benest and C.Froeschlé (eds.): "Perturbation Methods in Celestial Mechanics" Société Française des spécialistes de Physique to appear. (Proceedings of the Goutelas Spring School)

Following the example of the previous reports, this report does not claim to cover all fields of interest of Celestial Mechanics. A few particular topics which have seen recent developments and which were not covered in previous reports have been selected and reviewed by the specialists whose names appear as main contributors.

In the course of the report, Astronomy and Astrophysics Abstracts numbers are used when available. For the most common Journals the following abbreviations are used: CM for Celestial Mechanics, IC for Icarus, AA for Astronomy and Astrophysics, AJ for the Astronomical Journal, NA for Nature, APJ for the Astrophysical Journal and MNRAS for Mon.Not.R.Astron.Soc.. For the books and proceedings mentioned above we have used the following abbreviations: ACM for the proceedings of the conference "Asteroids, Comets, Meteors III", ASII for the book "Asteroids II", FBP for the proceedings of the Colloquium "Few Body Problem", PMCM for the book "Perturbation Methods in Celestial Mechanics". We use also the abbreviation ERAM for the volume 3 of the proceedings of the $10^{\text {th }}$ European Regional Astronomical Meeting (Šidlichovský ed., Praha 1987).

\section{B. Chaotic Behaviour of the Solar System}

(main contributor : Jacques Laskar)

The number of papers related to chaotic dynamics in celestial mechanics has increased so much in the last few years that a report on this subject would need much more space. The present report concentrates on chaotic motion in the solar system, while the introduction provides a brief outlook of the underlying theoretical works. 


\section{INTRODUCTION.}

After the work of Poincaré and Birkhoff, KAM theory (from Kolmogorov, Arnold and Moser) forms the background of most of the present work in Celestial Mechanics concerning hamiltonian systems close to integrable ones. For two degree of freedom hamiltonians, the invariant KAM tori separate the phase space inducing stability for all time. The breakdown of the $\mathrm{KAM}$ tori as the perturbation increases gives rises to large scale chaotic motion. Computation of the breakdown threshold of the invariant tori were numerically established by Chirikov on the basis of the overlap resonances criterion, and by Greene with the study of the stability of nearby periodic orbits (J.Math.Phys.20.1183). Other criterions and references can be found in (MacKay and Percival Commun.Math.Phys.98.469), (Celletti and Cherchia Commun.Math.Phys.118.119) and (Olvera and Simó Physica 26D.181).

Recently, in the case of the standard map, Lazutkin et al. (Physica 40D.235) derived an asymptotic formula for the angle of intersection of separatrices which is shown to be exponentially small with the perturbation. They also give an estimate of the size of the related chaotic zone. Related results have been obtained by Fontich and Simó (Ergod.Th.Dynam.Sys. 10.295 and 10.319).

In the regions where the invariant KAM tori do not survive, even in two degree of freedom systems, there exists a diffusion of the action like variables. But there are still invariants Cantor sets named cantori, which appear to form partial barriers. The diffusion rate through these barriers was studied by Mackay et al. (Physica 13D.55) and by Bensimon and Kadanoff (Physica 13D.82).

For systems of more than two degree of freedom, invariant tori and cantori do not confine the motion of the chaotic regions which is then subject to Arnold's diffusion and can take place in the Arnold web nearly everywhere in the phase space. Numerical studies on many degrees of freedom system were done by C. Froeschlé and Scheidecker in the early 70's. One of the essential tools in the study of the diffusion rate is the computation of the Lyapounov Characteristic Numbers (LCN) which was reviewed by C. Froeschlé (CM.34.95). Recent numerical studies of diffusion through cantori in 2-D systems and Arnold diffusion in 3-D systems are given in Contopoulos et al. (AA.172.55), Contopoulos and Barbanis (AA.222.329), and Marchal (46.022.189).

When the perturbation is small, Nekhoroshev's theorem (based on classical perturbation theory) show that the diffusion of the action is slow. This allows to obtain bounds on the variation of the actions over finite time which can be as long as the age of the universe (Benettin et al. CM.37.1, Giorgilli et al. CM.37.95, Lochak report LMENS.90.5 Ecole Normale Superieure, Paris, see also subsection 3.).

The numerical studies of chaotic behaviour need numerical integrations over a very long time or many iterations of a mapping. Hadjidemetriou (49.042.109) derived a semi-analytical mapping for 2-D systems near a resonance (see also Sun and Yan, CM.42.369), while Froeschlé and Petit (AA to appear) construct numerical synthetic mappings which can be used in general problems.

The reviews by Chirikov (Phys.Rep.52.263) and Hénon (Les Houches XXXVI, 1981) were very useful for the introduction of chaotic dynamics among astronomers. See also the book of Lichtenberg and Lieberman (Regular and stochastic motion, Springer Verlag, 1983), and the review of Escande (Phys.Rep.121.165), Percival (45.022.002), Simó (40.042.124 and PMCM to appear). The bock of drnold et al. (45.003.009) reviews the current developments of KAM theory, while the book of Itrowsmith and Place (An introduction to Dynamical Systems, Cambridge: (I.P, 19911) provide; introduction to the winthematics related to chaotic behaviour. 
For a review and references on Nekoroshev's theorem and adiabatic invariants, see the book of Lochak and Meunier (Multiphase Averaging for Classical Systems, Springer-Verlag, 1988). The chaotic behaviour in the solar system was reviewed by Wisdom (IC.72.241) and C. Froeschlé (ERAM.113, ACM.63)

\subsection{SATELLITES}

The chaotic tumbling of Hyperion was the first observable evidence of chaotic behaviour in the solar system (Wisdom et al. IC.58.137). Later on Wisdom showed that all irregularly shaped satellites must tumble chaotically before being captured into synchronous rotation (AJ.94.1350). Marciallis and Greenberg investigated the possible warming of Miranda during its chaotic rotation (NA.328.227). Celletti analysed the stability of several spin-orbit resonances in a 2 degree of freedom model by the effective construction of KAM surfaces trapping the motion (ZAMP.41.174). Ferraz-Mello, Dvorak and Karch (AA.179.304, CM.43.361) numerically studied chaotic motions in the $2 / 1$ resonance with Dione. Hénon and Petit (CM.38.67, IC.66.536, 49.042.126) analysed the interaction of two small satellites initially on very close circular orbits. Depending on the value of the impact parameter, different behaviour of the orbits take place. The transitions occur for a Cantor-like set of values of the parameter. The case of the Saturn coorbital satellites was analysed by Spirig and Waldvogel (40.042.029, 49.042.106). Extension to the elliptic case is undertaken by Liu and Sun (AA subm.). This problem is related to the scattering of particles coming from infinity and interacting with a dynamical system. In a similar way, Contopoulos (AA.231.41) studied orbits escaping to infinity in a galactic potential.

\subsection{URANIAN SATELLITES.}

Since Voyager II has revealed that the small satellites of Uranus (Miranda, Ariel and Umbriel) show evidence of melting, a considerable interest has developed in the history of the tidal evolution of these satellites during which they have possibly encountered several resonances.

Tittemore and Wisdom (IC.74.174) investigated the passage of Ariel and Umbriel through the $5 / 3$ resonance with the use of a mapping for the averaged planar elliptic RTBP (Restricted Three Body Problem). Dermott et al. (IC.76.295) use numerical integration to analyse the 3/1 Miranda-Umbriel resonance. In order to reduce the computing time, they increase the values of the mass of Umbriel and the $J_{2}$ of Uranus. They found chaotic motions with large increases in eccentricity and inclination which suggested a possible explanation for the high inclination of Miranda. This was explained by Tittemore and Wisdom (IC.78.63). Using the averaged circular inclined RTBP model through its associated mapping, they show that capture into a secondary resonance provides a mechanism for escape from the primary resonance which can produce the high inclination value of Miranda. Malhotra and Dermott (IC.85.444) confirmed and extended these results. They derived a "perturbed pendulum" model to understand the dynamics of the secondary resonances. The extent of the chaotic layers and locations of the secondary resonances were also confirmed by Henrard and Sato (CM.47.391) with a semi-analytical perturbative analysis.

Tittemore and Wisdom (IC.85.394) analysed with more complete models of 4 degree of freedom and the associated mappings the 3:1 Miranda-Umbriel, 5:3 Miranda-Ariel and 2:1 ArielUmbriel resonance. The analysis of different possibilities of passage into resonance allow them to derive a constraint on the tidal dissipation function $Q$ of Uranus.

\section{STABILITY OF LAGRANGIAN POINTS.}

The stability of the Lagrangian points in the Solar system has been revisited very recently. For the RTBP (Sun-Earth-Moon) the halo orbits near the libration points $L_{1}$ and $L_{2}$ are 
unstable periodic orbits. Simó et al. (44.042.054, ESA.SP-255, Acta Astro. 15.391) and Gomez et al. (ESOC report, 1987) computed precise orbits under the perturbation of a realistic solar system. The knowledge of the related stable and unstable manifolds allows them to develop a costless station keeping algorithm for these orbits. On the other hand, Giorgilli et al. (J.Diff.Eq 77.167) and Simó (Mem.R.Acad.Cien.Barcelona 48) use their version of Nekhoroshev's theorem to derive estimates for the effective stability (i.e. stability up to finite but long time) for the $L_{4}$ and $L_{5}$ points of the 3 degree of freedom spatial RTBP. Celletti and Giorgilli (CM subm.) improved these estimates in the application to the Earth-Moon and Sun-Jupiter systems. The stability of $L_{4}$ and $L_{5}$ was also numerically investigated by Zhang and Innanen (AJ.96.1983, AJ.96.1989, AJ.96.1995, FBP.141), and by El Bakkali (Thesis, 1990) in the framework of the near resonance theorem of Marchal (C.R.Acad.Sci.Paris II.306.505, 49.021.033). Analysis under the perturbation of a realistic solar system is made by Díez et al. (CM subm.)

\section{ASTEROIDS.}

The dynamics of the asteroids was reviewed recently by Nobili (ASII.862), C. Froechlé and Greenberg (ASII.827) for the mean motion resonances, Ch. Froeschlé and Scholl (CM.46.231, ERAM.125) and Scholl et al. (ASII.845) for secular resonances. Chaotic behaviour can also result from repeated close encounters. Milani et al. (IC.78.212) undertook extensive numerical integration of planet crossing asteroids over $200000 \mathrm{yr}$ with examples of chaotic motion. Apollo-Amor objects orbital evolution was studied by Wetherill (IC.76.563) and Olsson-Steel (AA.204.313). Franklin et al. (IC.79.223) found no stable orbits between Jupiter and Saturn over 107 years. With the analysis of the surface of section of the circular Hill problem, Chauvineau and Mignard (IC.83.360) derived stability conditions for binary asteroids. Murison (AJ.98.2346) investigated the RTBP model in order to find a scenario for the capture of an asteroid by Jupiter.

\subsection{ASTEROIDS MEAN MOTION RESONANCES.}

The understanding of the phase space of the $3 / 1$ resonance was achieved by Wisdom in his analysis by numerical integration of the averaged planar elliptic RTBP in the vicinity of the resonance (IC.63.272). The analysis of the chaotic zone in the surface of section gives the explanation of the sudden increase in eccentricity of the asteroids which can then cross the orbit of Mars or even of the Earth. This was also confirmed by direct numerical integration (NA.315.731).

Henrard and Lemaitre developed a semi-analytical perturbative treatment for two resonant arguments to generalize Wisdom's results to the $2 / 1$ resonance (CM.39.213, IC.69.266, ERAM.23) and to the 3/2 resonance (CM.43.91). In (IC.83.391) they identified the secondary resonances as the main generators of chaotic motion in the $2 / 1$ resonance and found the width and location of the chaotic zones in good agreement with the numerical results of Murray (IC.65.70) and Wisdom (IC.72.241). Henrard and Caranicolas adapted the same method to the analysis of the $3 / 1$ resonance (CM.47.99). The $3 / 1$ resonance was also analysed analytically by Koiller et al. (44.042.057) and by Neishtadt (45.42.008) who computed the diffusion of the adiabatic invariant due to successive crossing of the separatrix. Šidlichovsky investigated the 5/2 resonance (FBP.117) and Liu and Liao computed LCN in the averaged planar RTBP (50.098.096). Schubart found numerically non quasi-periodic behaviour for asteroids close to the $3 / 2$ and $4 / 3$ resonance (CM.43.309).

\subsection{SECULAR RESONANCES AND CHAOS.}

One important problem with the numerical study of the asteroid secular resonance is their very long time scale. Orbits need to be integrated over several million years before one is able 
to see any effect resulting from chaotic dynamics. Numerical investigations were made by $\mathrm{Ch}$. Froeschlé and Scholl by direct numerical integration over $1 \mathrm{Myr}$ to $4 \mathrm{Myr}$ of a test asteroid perturbed by Jupiter and Saturn (AA.166.326, AA.170.138, A A.179.294, CM.43.113). They found transitions between libration and circulation at $\nu_{16}$ and transitions from the resonance $\nu_{16}$ to $\nu_{6}$ which indicate chaotic behaviour. They also show (FBP.123) that 2335 James which is presently in the $\nu_{16}$ resonance, can enter the $\nu_{5}$ resonance. They discuss the possibility that the high inclination of the Appolo-Amor objects is due to overlapping of secular resonances. Sidlichovský (49.042.076, CM to appear) overcame the problem of long term numerical integration by the construction of a mapping for the secular hamiltonian reduced to the resonant part with $\nu_{5}$ and $\nu_{6}$ and found chaotic motion in the region where these two resonances overlap.

\section{COMETS.}

A general review of the dynamics of comets was given by Rickman and C. Froeschlé (CM.43.243). The use of Monte Carlo mappings in cometary dynamics was reviewed by $\mathrm{C}$. Froeschle and Rickman (CM.43.265). They stress that these methods only apply in the chaotic regions. This was confirmed by Baille and C. Froeschle (AA to appear) with the application of the method to the standard mapping.

\subsection{ORIGIN OF THE SHORT PERIOD COMETS.}

The capture scenario of the nearly parabolic comets coming from an isotropic Oort cloud presents two difficulties: the probability of capture is too small and the distribution of the inclinations does not fit the observations (Bailey NA.345.21). This question was investigated by Duncan et al. (APJ.328.L69, APJ.355.667) who support the hypothesis of SP comets coming from a transneptunian belt of low inclination (Kuiper belt). Investigations were conducted by numerical integration with a planar circular model for the outer planets with an increase of their masses in order to accelerate the process. They also used a mapping for the circular RTBP (IC.82.402) to study the stability of possible bands of residual planitesimals from the protoplanetary disk in the outer solar system. The stability of a comet disk beyond Neptune was analysed by Torbett and Smoluchowski (NA.345.49).

\subsection{LONG PERIOD COMETS.}

Petrosky (42.102.035) and nearly at the same time Sagdeev and Zaslavsky (44.102.040) constructed analytically a mapping (the Keplerian map) for the motion of near parabolic comets perturbed by Jupiter. The motion was found to be chaotic with a chaotic zone extending to the Oort cloud. It was compared to numerical integration by Petrosky and Broucke (CM.42.53). Chirikov and Vecheslavov (AA.221.146) fitted the parameters of a similar Keplerian map to the observational data to show that the motion of comet Halley is chaotic and that prediction is lost after 29 revolutions. This was confirmed by C. Froeschlé and Gonczi by the computation of the LCN of comet Halley (CM.43.325) and by Dvorak and Kribbel (A A.227.264). Natenzon et al. (Phys.Lett.A.145.255) generalized this study to long period comets for all values of the eccentricity. They show that a parabolic or moderately hyperbolic comet can be temporarly captured during a long time in resonance with Jupiter.

\section{THE CHAOTIC MOTION OF THE PLANEITS.}

In the last 3 years, our vision of the motion of the planets has thanged. Numcrical integra

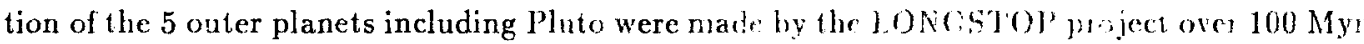
(Carpino et al. AA.181.182, FBP.165, Nobili et al . A A.210.313: l:13P.14i) and at MT vith 
the Digital Orrery over 200 Myr (Appelgate et al. AJ.92.176). These integrations were very interesting for our understanding of the dynamics of the outer planets but did not reveal any strong evidence of chaotic motion (like positive LCN) although the difficulties in the reduction of the output of LONGSTOP1B to quasi periodic form suggest a non regular pattern at the level of $10^{-4}$ in the eccentricity of Saturn (Nobili, CM.45.293). When extending their numerical integration with the Orrery up to $845 \mathrm{Myr}$, Sussman and Wisdom found a positive Lyapounov exponent of $1 / 20 \mathrm{Myr}$ for Pluto indicating that its long term evolution is chaotic (Science, 241.433). Discussion about the possible origin of this chaotic behaviour was made by Milani et al. (IC.82.200). Laskar uses a semi-analytical approach with the numerical integration of the averaged system of second order for the 8 main planets (AA.144.133, AA.157.59, AA.198.341): integrations over $30 \mathrm{Myr}$ revealed the existence of secular resonances among the inner planets (ERAM.95); the extension of the integration up to $200 \mathrm{Myr}$ show a positive LCN of $1 / 5 \mathrm{Myr}$ which indicates a chaotic behaviour for the solar system (NA.338.237). The size of the chaotic region was estimated numerically by the variation with time of the fundamental frequencies which appear to be relatively large in the directions related to the inner planets proper modes (IC to appear).

\section{Celestial Mechanics for Stellar Systems (main contributor : D.C. Heggie)}

Stellar dynamics shares with celestial mechanics the same equations of motion and a broad range of concerns, e.g. periodic orbits, potential motion, stability, bifurcations, etc. Unfortunately few researchers have interests in both fields, and so remarkably little cross-fertilisation has taken place. The following report mentions some topics in stellar dynamics which should be of particular interest to members of this commission.

\section{THE 3-BODY PROBLEM.}

Today the widest range of applications of the three-body problem is in stellar astronomy (45.012.051, 46.042.019, Valtonen and Mikkola , Annu.Rev.Astron.Astrophys. to appear). On the other hand it is becoming increasingly clear that the point-mass approximation is inadequate for some applications; therefore it is sometimes necessary to know something about the minimum distances between the participating stars (46.118.050). Coallescence can occur (45.151.007 \& $110,49.151 .065,93.31124)$, and it is even becoming possible to model triple systems with 'stars' of finite size (93.50438).

One area where developments in celestial mechanics can have a great impact is in methods for the computation of perturbed few-body orbits (45.021.019). Indeed improved methods of regularisation have been developed recently (Mikkola and Aarseth CM.47.375).

The problem of (temporary) capture is an old one in celestial mechanics, and is of considerable importance in stellar dynamics too (49.151.092). Relatively long-lived triple systems formed by capture are usually hierarchical, and perturbations from the outer companion may have important effects on the mass-transfer rate between the inner pair (45.117.208). Perturbations of binary orbits by the galactic tide are also of interest (46.151.024). On the other hand, study of chaotic three-body motions in which all distances are comparable has applications to the dynamics of observed triple galaxies (49.151.058).

Perturbations by a relatively distant intruder can also be used to study the scattering problem, i.c. encounters between a binary star and an incoming third body, though semi-numerical 
work is desirable because of the strength of the perturbations (44.042.034 \& 063). In addition to details of the perturbations during a single scattering encounter, it is even more important (in stellar applications) to know the statistical distribution of these changes (45.151.100). An example is the effect of encounters on binary pulsars in globular clusters (92.144.836), where the rates of exchange, disruption, and change in eccentricity are important. The special methods developed in celestial mechanics for the study of close triple approaches can be of value in the theoretical study of these statistical problems (45.042.065, Heggie and Sweatman MNRAS subm.); close approaches are important in the ejection of 'runaway stars' from young clusters (46.151.001, 93.70944). There is increasing interest in four-body encounters (binary-binary scattering), and the mass-dependence of three-body scattering (49.151.001), from these points of view.

\section{ORBIT THEORY.}

This is the other main concern shared by celestial mechanics and stellar dynamics, and chapter 3 of 44.003 .028 is an excellent introduction. Actually the three-body problem also provides an appropriate perspective. It turns out that properties of the orbits in the restricted problem are a useful guide to the behaviour of orbits in the potential of a rotating barred galaxy (49.151.105, 93.78338). This is true, for example, with regard to escape orbits in barred potentials (45.151.102), and the concept of zero-velocity surfaces can obviously be extended to binary galaxies (49.151.094).

Within the field of dynamical systems fully integrable systems may seem too special and too simple to be of further interest. But the existence of integrals of the motion is of crucial importance in stellar dynamics, because of their role in Jeans' theorem. In fact integrable potentials (Stäckel potentials) provide an excellent guide to more realistic potentials, and are a good model for them (de Zeeuw, Ann.N.Y.Acad.Sci. 536.15, 46.155.164).

As in celestial mechanics the study of periodic orbits and their stability and bifurcations has an important place in the theory of general galactic potentials (45.042.043, 46.151.042, 49.151.104), but for not quite the same reasons. Stable periodic orbits in many realistic nonrotating potentials are surrounded by large regions of approximately quasi-periodic orbits, and so provide a basis for classifying most orbits; indeed methods have been developed to trace the approximate invariant tori of these orbits numerically (McGill and Binney MNRAS subm.). But, in addition, the distinctive importance of periodic orbits in stellar dynamics is that they give suitable loci for the occurrence of gas $(46.151 .098,92.144909)$, because there are no near self-intersections.

One area where the exploration of periodic orbits is still developing strongly is in the study of three-dimensional orbits in rotating potentials $(46.151 .053,49.151 .009)$. One goal of current research should be to provide for these potentials the rather complete kind of qualitative understanding of orbit structure which we now possess for non-rotating potentials.

Resonances are of considerable importance in galactic dynamics, just as in the solar system, but there are again distinctive features of the stellar dynamical problem. The task of forming self-consistent models of barred galaxies places special emphasis on the 4:1 resonance, for example (46.151.007 \& 055). Resonant integrable model potentials are a useful tool for such studies (Contopoulos Ann.N.Y.Acad.Sci. 536.1, 49.151.100).

There have been a number of papers investigating the extent of chaotic orbits in various galactic models. Among the various causative mechanisms that have been explored are (i) a black hole or some other singularity in the potential (Pfenniger and de Zeeuw in Dynamics of Dense Stellar Systems Cambridge U.P. 1989, 49.151.063), (ii) the time-dependent potential of a 
companion galaxy (93.58210), and (iii) the rotation of a non-axisymmetric potential (45.151.093, Martinet and Udry AA to appear), which once again underscores the topicality of this problem. Stochasticity can be effective in heating (thickening) a galactic disk, and can be described by a Fokker-Planck equation (45.151.005). Gurzadyan \& Kocharyan (46.155.072) have given an estimate of the divergence of neighbouring orbits (inverse Liapounov characteristic exponent) which is surprisingly short in galactic terms. Chaos can also arise in the more subtle models used for the evolution of globular star clusters, and are susceptible to the usual methods for studying strange attractors (Breeden et al. Astrophys.J subm.).

Finally it is worth mentioning one aspect of galactic orbit theory which is not Hamiltonian. In certain important circumstances the response of a galaxy to a moving object (such as a globular cluster) can be modelled as an additional friction force (dynamical friction). Recent studies of orbits with friction include $45.151 .020,107 \& 148$, and $46.151 .017 \& 086$, but perhaps the most beautiful application has been to the origin of faint "shells" which are observed in so many elliptical galaxies (46.151.029). On the other hand there is evidence that the response of the galaxy is more subtle than the concept of dynamical friction would suggest $(49.151 .045$, 46.151.049).

In conclusion, it can be seen that celestial mechanics and stellar dynamics have much in common, though there are some differences of style. In stellar dynamics there is greater emphasis on applications, and less emphasis on careful mathematics. On the other hand the richness and diversity of phenomena within the field of stellar dynamics ensures that there is much scope for the powerful and well developed methods of celestial mechanics.

\section{Secular Resonances in the Asteroid Belt}

(main contributor : Andrea Milani)

The state of the art on this subject, as reported at the last IAU Assembly (Froeschle and Scholl CM.46.231), was as follows. The location of the main secular resonances $\nu_{5}$ (perihelion locked to the one of Jupiter), $\nu_{6}$ (perihelion locked to the one of Saturn) and $\nu_{16}$ (node locked with the node of Saturn) in the asteroid belt had been computed within a nonlinear theory of secular perturbations (Williams and Faulkner IC.46.390). The behaviour of an orbit close to one of these resonances could be described by a semianalytic model (Nakai and Kinoshita CM.36.391, Kozai in Resonances in the motion of planets, satellites, asteroids Univ. de S.Paolo 1985, Yoshikawa CM.40.233). All these results were obtained by combining numerical averaging with respect to the fast variables (the anomalies of both the asteroid and the perturbing planets) with some simplifying assumption only approximately satisfied by the real problem, e.g. by neglecting the inclination of the perturbing planets and/or the changes in some action variable. An extensive campaign of numerical integrations (Froeschlé and Scholl AA.166.326, AA.170.138, AA.179.294) showed that secular resonances are not only singularities in the analytical procedure to compute a long term orbit, they are real and very effective mechanism to increase the eccentricities of inner belt asteroids to Earth-crossing values, as it had been suggested (Wetherill and Williams in Origin and distribution of the elements (Ahrens ed.) Pergamon 1979). However, the numerical integrations were performed with a simplified model (containing only the planets Jupiter and Saturn), and the semianalytical theories were obtained with somewhat ad hoc procedures and unsafe approximations.

Significant progress has occurred in the last few years, both in the theory and in the understanding of the astrophysical consequences of secular resonance. The topology of the secular 
resonant motion around $\nu_{6}$, more or less understood from the previous computations, has been investigated in a purely analytical way and found to coincide with the second fundamental model of resonance (Šidlichovský 1989 preprint). The cases of $\nu_{16}$ and $\nu_{5}$ are more difficult, because the inclinations are too large to allow the use of a truncated expansion of the perturbing function, and their investigation by numerical integration has continued (Froeschlé and Scholl AA .227.255 and in FBP). However the interpretation of the numerical experiments is not obvious; where the resonances $\nu_{5}$ and $\nu_{16}$ overlap, alternation between different libration occurs together with all the well known signatures of chaotic behaviour (Froeschlé and Scholl CM.46.231, Śidlichovský CM to appear); moreover the numerical experiments provide osculating orbital elements, which have somehow to be transformed into proper elements for comparison with a theory.

As a corollary of the new theory of proper elements (Milani and Kneževic̀ CM to appear), a new analytic theory of the location of the secular resonances was derived taking into account the terms of degree four in the eccentricities and inclinations of both the asteroid and the perturbing planets (Jupiter and Saturn). The second order effects resulting from nearby mean motion resonances were also accounted for, up to degree two; this results in a significant bending of the secular resonance surfaces, e.g. near the 3:1 gap. Also the use of more up to date values of the fundamental frequencies resulted in a displacement of the estimated position of the $\nu_{6}$ resonance, which is now believed to be very close to the densely populated Flora region, hence to be an even more relevant source of meteroites. New secular resonances of higher degree, involving combinations of four fundamental frequencies, can also be studied; one such resonance crosses the Eos family. The mutual interaction of the secular and mean motion resonances is understood in special cases, e.g. in the 2:1 resonance (Lemaitre and Henrard IC.83.391), while other cases such as the 5:1 resonance are much more difficult (Froeschlé and Scholl CM.43.113, Milani et al. IC to appear)

The semianalytic method has been revised by using a fully canonical transformation formalism, with rigorous averaging in a way matched to the topology of the libration behaviour (Morbidelli and Henrard CM subm.). The location of the secular resonances surfaces has been computed without truncation of the high powers of the eccentricity and the inclination, and also taking into account the second order near-resonant effects up to degree six. The behaviour for the resonant and near-resonant orbits is described by a local model, which takes into account of the effect of arguments different from $\omega$ as perturbations to the Williams-type model. This allows a reliable computation of the location of the resonant surface, which improves some previous computations (Yoshikawa CM.40.233, Milani and Kneževic̀ CM to appear). The dynamical behaviour in the neighbourhood of the three main secular resonances is also investigated by the averaging method (Morbidelli and Henrard CM subm.); for the $\nu_{6}$ resonance the comparison with numerical integration gives a good confirmation of the theory, but for the $\nu_{5}$ resonance there are significant problems. The case with libration of $\omega$ is also investigated in this paper. There is a group of asteroids which exploit the effects of either libration or uneven circulation of $\omega$ to avoid close approaches with the Earth (Froeschle and Scholl AA.170.138, Milani et al. IC.78.212); the same mechanism contributes to the dynamical stability of Pluto (Milani et al. IC.82.200).

A subject which is just beginning to be investigated is the possibility of secular resonance sweeping in the primordial solar system (Lemaitre and Dubru CM subm.); if the dynamical configuration of the bulk of the mass orbiting around the Sun changes, very large changes in the resonances could result in temporary captures and could explain the pumping up of eccentricities and inclinations for the asteroids (and may be the planets a: well). 


\section{E. Rotation of Celestial Bodies in the Solar System}

(main contributor : Hiroshi Kinoshita)

With the introduction of new techniques (VLBI, radar ranging, laser ranging) and the observations from space crafts our knowledge about the rotational states of the bodies of the solar system has increased considerably in the last decade. In many instances we can no longer investigate the orbital motion and the rotational motion independently but we have to take into account their coupling. On the other hand a better knowledge of the rotational states may provide some clues about the formation and dynamical evolution of the Solar System.

\section{NUTATION OF THE EARTH.}

In the reduction of rotational data of the Earth, the IAU 1980 Nutation Series are being used. The series are based on Kinoshita's (20.042.046) rigid Earth theory and then modified by Wahr's theory (29.081.005) which uses a model of an elastic and oceanless Earth with a fluid core. The accuracy of this nutation series is at the one mas (milli-arcsecond) level, which is enough for the classical optical observations. However, recent observational accuracy of VLBI has reached the 0.1 mas level, and detected systematic residuals from the present IAU 1980 Nutation Series and out of phase terms in nutations which originate from the dissipative mechanism in the core-mantle boundary. Also 20 years of LLR (lunar-laser ranging) data which cover one period of the nodal motion of the Moon can now separate precession and nutation at the mas level or better. Both VLBI and LLR observations suggest systematic differences of amplitude in the main nutational terms. Under this circumstance, the IAU Working Group on the theory of Nutation recommends to develop a new theory of nutation including all known effects at the 0.1 mas level. Kinoshita and Souchay (CM to appear) have reconstructed the nutation series for a rigid Earth model with use of ELP2000 (Chapront-Touzé and Chapront 34.094.002) and VSOP82 (Bretagnon 32.042.038) for the Moon and the Sun respectively, instead of Brown's theory of the Moon and Newcomb's theory of the Sun that were used in the old nutation series. The new theory includes both direct and indirect planetary perturbations and second order perturbations. One of the significant change in the new series arises from the coupling effect between the rotational motion and the orbital motion. Souchay and Kinoshita (CM subm.) have compared the new theory with numerical integration and found the residuals to be of the order of 0.04 mas. Non-rigid effects have been computed by Wahr and Bergen (42.081.018), Zhu et al (AJ.99.1024) and Dehant (Geoph.J.R.A.S. 110.477). In spite of these considerable improvements in theoretical computations, the new nutation series still cannot explain residuals detected by VLBI and LLR. Perhaps this indicates that the theoretical calculations (rigid or nonrigid or both) are still incomplete.

The accuracy of 0.1 mas corresponds to $0.3 \mathrm{~cm}$ displacement on the surface of the Earth. One may suspect that such an accuracy may not be necessary to discuss the solar system dynamics. However this accuracy (or better) is necessary to realize the relativistic reference frames in which the motions of celestial bodies are described.

Kubo (CM subm.) derives equations of motion of a deformable body in a Hamiltonian form with use of Andoyer variables and applies them to the nutation of the Earth with an elastic mantle. This formulation may be applicable to the nutations of Jupiter, Saturn, Uranus and Neptune, which may be detected in the future.

\section{EARTH-MOON SYSTEM.}

The rotational angular momentum of the Earth is of the same order than the orbital angular momentum of the Earth-Moon system. Therefore, in order to study the dynamical evo- 
lution of the Earth-Moon system we have to take into account the coupling effects between the rotational motion and the orbital motion. After the pioneering works of Munk and MacDonald (Rev.Geophys.Space Phys. 2.467) and Goldreich (66.8382, Rev.Geophys. 4.411) the research in this field progressed slowly. Recent works are reviewed by Boss and Peale (43.094.032). These researches show the the orbital inclination increases toward the past. Recently Webb (Geophys.J.R.Astron.Soc.61.573) and Ooe et al. (AGU Monograph to appear) have shown that the position of continents has a great effect on the tidal dissipation : it indicates that in the discussion of the history of the Earth-Moon system, plate-tectonic motion should be included. This is an important problem. If the orbital inclination does not increase toward the past, the capture theory which is one of the theories of the Moon's origin is in trouble.

\section{OTHER PLANETS.}

The present theory of a rigid nutation can be applied to Mercury, Venus and Mars which can be treated approximately as rigid bodies, since the present observational accuracy cannot detect the non rigid effects. Nutations of the figure axis which are observable are composed of two components: Poisson terms and Oppolzer terms, both of which are forced periodic perturbations. The amplitude of the Oppolzer terms is approximately inversely proportional to $\left(\omega-n_{\nu}\right)$, where $\omega$ is the spin rate of the Earth and $n_{\nu}$ is the angular speed of the forced perturbation while the amplitude of the Poisson terms is inversely proportional to $n_{\nu}$. Therefore Oppolzer terms of the Earth and Mars are very small compared with Poisson terms since $\omega \gg n_{\nu}$. On the other hand, Oppolzer terms of Mercury and Venus the rotation periods of which are very slow are comparable to Poisson terms. So far there are no theory of Nutation for Mercury and Venus.

The present rotation of Venus is retrograde, which is unique in the solar system. Four classes of dynamical histories are presented to account for the slow and retrograde rotation of Venus : (a) the loss of an satellite, either destroyed by impact or escaped (becoming Mercury ?), (b) Venus has evolved since its formation from a rapid to a slow rotation rate under the influence of solar tidal torques, (c) Venus originated with a retrograde rotation, (d) Venus evolved from direct to retrograde rotation. The references on these scenarios are found in Lago and Cazenave (26.093.028) and Peale (CM.46.253). As for the scenario (d), using the hypothesis that only solar tidal torques and core-mantle coupling have been active since the formation of Venus, Lago and Cazenave (26.093.028) find quite conceivable that Venus had originally a rotation similar to the other planets and has evolved in $4.5 \times 10^{9}$ years from a rapid and direct rotation (12-hour spin period and nearly zero obliquity) to the present slow retrograde one. With the use of similar models as Lago and Cazenave (26.093.028), Shen and Zhang (49A.093.025) show that Venus has evolved from a spin period between 7 hours and 2 days and an obliquity of about $90^{\circ}$. Scenario (c) was investigated by Giuli (68.3802,IC.8.301), Harris (19.107.012), Tanikawa et al. (49A.042.074). Within the framework of the planar restricted three-body problem, Tanikawa et al. (49A.042.074) showed that there exists a value $e_{0}$ of the eccentricity of a protoplanet such that it acquires a negative angular momentum through collisions with planetesimals with a smaller eccentricity. It is worthy of extending this investigation from the two dimensional to the three dimensional problem in order to discuss the origin of the obliquities (around $90^{\circ}$ ) of Uranus and Pluto.

Mercury (which is in a spin-orbit resonance and in a Cassini state) is an interesting planet in every aspect of its dynamics. Peale (49A .092.019) gives a comprehensive review on the dynamics of this planet. Physical data on Mercury are gradually increasing, which will give some constraints on its dynamical evolution and the late stages of its formation.

The spin axis of Uranus and Pluto are inclined respectively $98^{\circ}$ and $122^{\circ}$ to their orbital planes. An impact of a large planetesimal in the late stages of accretion might explain these large 
tilts. However, it would be more satisfactory to find an explanation based upon the dynamical evolution of the planets and their satellites rather than to rely on catastrophic events in the primordial stages.

\section{ASTEROIDS AND COMETS.}

Data on rotational periods are available for about 400 asteroids (Binzel et al. ASII.416) and 47 comets (Whipple 32.102.017) and data on the orientation of the spin axis are available for 30 asteroids (Magnusen et al. ASII.66) and 12 comets (Sekanina 30.102.010). These numbers are growing slowly but are still small for statistical discussions on the origin of families or on the formation and dynamical evolution of the Solar System.

Recent observations of Halley from both space crafts and ground have revealed that Halley has two periods : it indicates that the spin axis does not coincide with the figure axis. Whether the rotation of Halley is in the short axis mode (secularly stable) or in the long axis mode (secularly unstable) is still controversed (Peale and Lissauer 49A.103.145, Abergel and Bertaux IC.86.21, Belton IC.86.30). A long axis mode of rotation could be an indication that the rotational state of comets is not primordial. Anyhow Halley with a large triaxiality under various forces gives us a challenging problem on rotational dynamics.

\section{NATURAL SATELLITES.}

Among the natural satellites other than the Moon, Phobos and Hyperion are the only ones for which the observational data available allows an analytical discussion. From hundreds of high-resolution images obtained by the Viking Orbiters over a 4-year period from 1976 to 1980 , Duxbury and Callahan (49A.097.139) found a forced libration amplitude of $0 .^{\circ} 8$ and determined the inertial orientation of the spin axis. Borderies and Yoder (AA.233.235) constructed the rotation theory of Phobos the series cutoff of which is about 0.3 mas or $5 \mathrm{~cm}$ displacement on the surface of Phobos. This theory together with the secular motion of the pericenter of Phobos gives a constraint on the principal moments of inertia.

Wisdom et al. (37.100.050) predicted that Hyperion is in a state of chaotic rotation. With use of a light curve obtained by a CCD camera, Klavetter (49A.100.001) showed that Hyperion is not in any periodic rotation state, thus strongly suggesting it is chaotic. By changing the dynamical model of Wisdom et al. to fit the light curve, Klavetter (A.J. 98.1855) estimated the initial conditions of the rotation and the ratios of the principal moments of inertia. From the principal moments of inertia together with the topographical data, we can estimate the mass distribution of a satellite.

Jacques Henrard

President of the Commission. 